\title{
Lipoprotein Receptor-related Protein 6 Signaling is Necessary for Vasculogenic Differentiation of Human Dental Pulp Stem Cells
}

\author{
Gleyce O. Silva, DDS, MS, PhD, ${ }^{*}{ }^{\dagger}$ Zhaocheng Zhang, MD, PbD, * Carolina Cucco, DDS, MS, PbD, * \\ Min Ob, BS, * Carlos H.R. Camargo, DDS, MS, PhD, ${ }^{+}$and Jacques E. Nör, DDS, MS, PhD ${ }^{* \neq J \|}$
}

\section{Abstract}

The aim of this study was to evaluate the effects of Wnt signaling through lipoprotein receptor-related protein 6 (LRP6) and Frizzled6 on the endothelial differentiation of dental pulp stem cells (DPSCs). DPSCs were stably transduced with enhanced green fluorescent protein (EGFP)-tagged lentiviral vectors (short hairpin RNA-LRP6, short hairpin RNA-Frizzled6, or empty vector controls). We evaluated the effects of LRP6 and Frizzled6 on expression of endothelial markers and on capillary tube formation mediated by DPSCs induced with recombinant human Wnt1 (rhWnt1) and/or recombinant human vascular endothelial growth factor $_{165}$ (rhVEGF $_{165}$ ). In vivo, tooth slices/ scaffolds were seeded with LRP6-silenced, Frizzled6silenced, or vector control DPSC cells and transplanted into immunodeficient mice. The density of blood vessels generated by DPSCs differentiated into vascular endothelial cells was analyzed by immunohistochemistry for EGFP. The rhWnt1 and rhVEGF $_{165}$ induced expression of active $\beta$-catenin in control DPSCs and in Frizzled6-silenced DPSCs, but not in LRP6-silenced DPSCs. Furthermore, VEGF and interleukin-8 were downregulated in LRP6-silenced DPSCs, but not in control DPSCs or in Frizzled6-silenced DPSCs $(P<.05)$. Likewise, rhWnt1 and rhVEGF$_{165}$ induced expression of the endothelial marker VEGF receptor-2 in control DPSCs and in Frizzled6-silenced DPSCs, but not in LRP6-silenced DPSCs. These data correlated with a trend for lower density of capillary sprouts generated by LRP6-silenced DPSCs when compared with control DPSCs in Matrigel. In vivo, tooth slice/scaffolds seeded with DPSC-short hairpinRNA-LRP6 cells showed lower density of human blood vessels (ie, EGFP-positive blood vessels), when compared with tooth slice/scaffolds seeded with vector control cells $(P<.05)$. Collectively, these data demon-

strated that LRP6 signaling is necessary for the vasculogenic differentiation of human DPSCs. (J Endod 2017;43:S25-S30)

\section{Key Words}

Angiogenesis, cell fate, regenerative endodontics, tissue engineering, Wnt

D ental pulp tissue engineering can be achieved with the use of a scaffold that provides a physiological three-dimensional microenvironment that is conducive to stem cell adhesion survival, and differentiation in presence of adequate morphogenic signaling molecules $(1,2)$. Successful dental pulp tissue regeneration also requires the fast establishment of a functional vascular network that is able to supply the tissue with oxygen, nutrients, and immune cells while removing by-products and waste (3). Dental pulp stem cells (DPSCs) have the ability to differentiate into odontoblasts, osteoblasts, fibroblasts, adipocytes, neural-like cells, and vascular endothelial cells (4-6). Although DPSCs have potential to differentiate into endothelial cells, the molecular events regulating this process are not fully understood.

Wnt signaling plays a major role in the regulation of cell fate decisions during development $(7,8)$. In absence of Wnt, a multi-protein complex including glycogen synthase kinase 3 beta (GSK-3 $\beta$ ) constantly phosphorylates $\beta$-catenin, signaling its degradation (9). Activation of Wnt signaling results in the translocation of $\beta$-catenin to the nucleus where it interacts with T-cell factor/lymphoid enhancer factor (TCF/ LEF) family proteins to modulate expression of several downstream genes, including several proangiogenic factors such as vascular endothelial growth factor (VEGF), interleukin-8 (IL-8), and vascular endothelial growth factor receptor 2 (VEGFR2) (10-12). Wnt signaling involves a complex set of receptors that include the Frizzled family and low-density lipoprotein receptor-related protein (eg, LRP5/6). Frizzled functions as a $G$ protein coupled receptor that preferentially couples to $\mathrm{G} \alpha_{\mathrm{s}}$ heterotrimeric $\mathrm{G}$ proteins (13). More direct evidence for a role of $\mathrm{G}$ proteins in Wnt pathway activation comes from reconstitution studies showing that several of them have the capacity to inhibit $\beta$-catenin phosphorylation by GSK-3 $\beta$.

From the *Department of Cariology, Restorative Sciences and Endodontics, University of Michigan School of Dentistry, Ann Arbor, Michigan; ${ }^{\dagger}$ Department of Restorative Dentistry, Institute of Science and Technology, São Paulo State University, São José dos Campos, São Paulo, Brazil; ${ }^{\ddagger}$ Comprehensive Cancer Center, University of Michigan, Ann Arbor, Michigan; ${ }^{\S}$ Department of Biomedical Engineering, University of Michigan College of Engineering, Ann Arbor, Michigan; and "Department of Otolaryngology, University of Michigan School of Medicine, Ann Arbor, Michigan.

Address requests for reprints to Prof Jacques E. Nör, Biomedical Engineering, Otolaryngology, University of Michigan, 1011 N University Room 2309, Ann Arbor, MI 48109-1078. E-mail address: jenor@umich.edu

0099-2399/\$ - see front matter

Copyright (c) 2017 American Association of Endodontists. http://dx.doi.org/10.1016/j.joen.2017.06.006 


\section{Regenerative Endodontics}

For example, it has been proposed that $\mathrm{G} \beta \gamma$ promotes the recruitment of GSK-3 $\beta$ to the plasma membrane to enhance LRP6 phosphorylation and activation (14).

Studies have found that $\mathrm{Wnt} / \beta$-catenin signaling plays a role in vasculature in vivo and in the differentiation of stem cells into odontoblasts, endothelial cells, osteoblasts, and neural cells (5, 15-19). Here we evaluated the role of Wnt receptors on the differentiation of DPSCs into endothelial cells. We hypothesize that LRP6 and Frizzled6 regulate Wnt-induced differentiation of DPSCs into endothelial cells.

\section{Cell Culture}

\section{Materials and Methods}

DPSCs (20) were cultured in minimum essential medium Eaglealpha modification (alpha-MEM) (Invitrogen, Carlsbad, CA) supplemented with $15 \%$ fetal bovine serum (Invitrogen) and 1\% penicillin/ streptomycin (Invitrogen) at $37^{\circ} \mathrm{C}$ and $5 \% \mathrm{CO}_{2}$. Endothelial differentiation was induced by culturing DPSCs with endothelial cell growth medium (EGM2-MV; Lonza, Walkersville, MD) supplemented with $50 \mathrm{ng} / \mathrm{mL}$ recombinant human $\mathrm{VEGF}_{165}\left(\right.$ rhVEGF $\left._{165}\right)$ (R\&D Systems,
Minneapolis, MN) and/or 50 ng/mL rhWnt-1 (Cell Sciences, Canton, MA). Endothelial differentiation was assessed in vitro by determining the expression of endothelial markers by Western blot. Human dermal microvascular endothelial cells (HDMECs) (Lonza) were used as positive control and cultured in EGM2-MV. Culture medium was changed every 2 days in all experiments included here.

\section{LRP6 and Frizzled6 Gene Silencing}

Gene silencing was performed with enhanced green fluorescent protein (EGFP)-tagged lentiviral vectors encoding short hairpin RNA (shRNA) constructs, as described previously $(5,6)$. The 293T cells were transiently cotransfected with lentivirus packaging vector psPAX2, pMD2.G, and ShRNA-C (control), shRNA-LRP6, or shRNAFrizzled6 (Vector Core, University of Michigan, Ann Arbor, MI) with calcium phosphate. Supernatants containing lentiviruses were used to infect DPSCs overnight. Transduced DPSCs were selected by exposure to $1 \mu \mathrm{g} / \mathrm{mL}$ puromycin (InVivogen, San Diego, CA) for at least 1 week. Infection efficiency was determined by fluorescence and gene silencing by Western blot (Fig. $1 A$ and $B$ ).
A

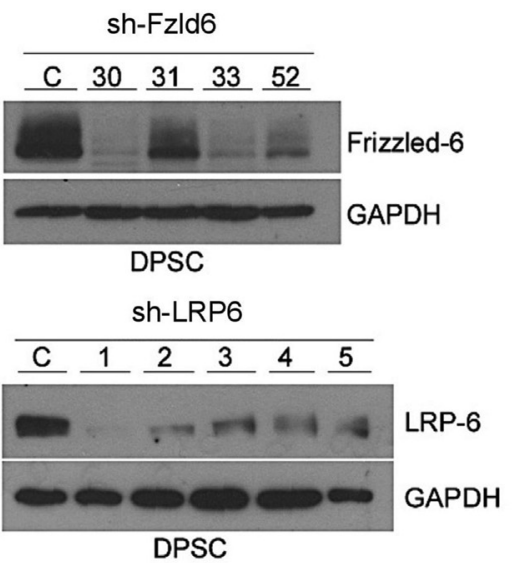

B
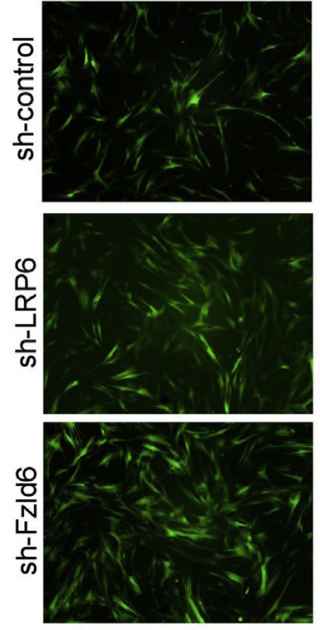

C

\begin{tabular}{|c|c|c|}
\hline \multicolumn{3}{|c|}{ VEGF (ng/ml) } \\
\hline sh-control & sh-LRP6 & sh-Fzld6 \\
\hline
\end{tabular}

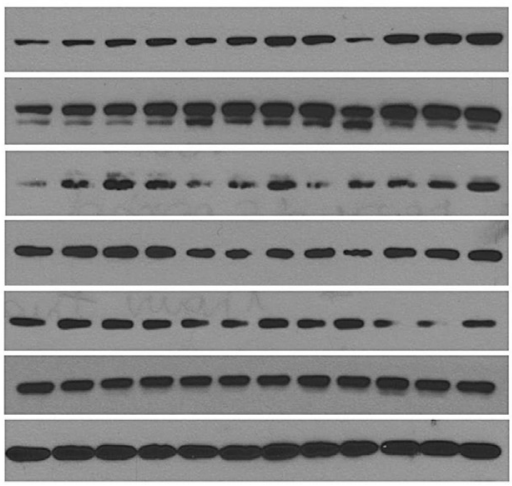

Figure 1. Effect of LRP6 and Frizzled6 on VEGF or Wnt1 signaling in DPSCs. $(A)$ Western blots to evaluate effectiveness of LRP6 or Frizzled6 silencing in DPSCs. We tested several shRNA sequences for Frizzled6 (\#30, 31, 33, 52) and for LRP6 (\#1-5). (B) Fluorescence image to evaluate effectiveness of lentivirus-mediated transduction of shRNA-LRP6 or shRNA-Frizzled6 into DPSCs. All lentiviral vectors used here (including controls) contained EGFP. Photomicrographs were taken at $\times 200$ magnification. $(C)$ Western blots depicting effect of increasing concentrations of rhVEGF165 or rhWnt1 on phosphorylated and total $\beta$-catenin, GSK-3 $\beta$, and AKT. 


\section{Western Blot}

Cells were lysed in NP40 (nonyl phenoxypolyethoxylethanol) buffer; proteins were electrophoresed in sodium dodecylsulfate-polyacrylamide gel and transferred to nitrocellulose membranes (Protran; Whatman, Dassel, Germany). Membranes were incubated at $4^{\circ} \mathrm{C}$ overnight with primary antibodies as follows: mouse anti-active or anti-total $\beta$-catenin, mouse anti-phospho and anti-total GSK-3 $\beta$, mouse antiphospho and anti-total AKT, rabbit anti-VEGFR1, rabbit anti-VEGFR2, rabbit anti-LRP6, rabbit anti-Frizzled6, mouse anti-GAPDH, and antiB-actin. SuperSignal West Pico chemiluminescent substrate (Thermo Scientific, Rockford, IL) was used to visualize immunoreactive proteins.

\section{Enzyme-linked Immunosorbent Assay}

Conditioned medium generated by DPSCs for 24 hours was collected and used for enzyme-linked immunosorbent assay (ELISA) by Quantikine Colorimetric Sandwich ELISAs (R\&D Systems), according to manufacturer's instructions. Supernatant was added to each well of a 96-well plate containing anti-VEGF or anti-CXCL8 antibody. Optical density was analyzed in spectrophotometer $(450 \mathrm{~nm})$.

\section{Gapillary Sprouting Assay}

We cultured $5 \times 10^{4} /$ well DPSCs with endothelial cell growth medium (EGM2-MV; Lonza) supplemented with $50 \mathrm{ng} / \mathrm{mL} \mathrm{rhVEGF}_{165}$ (R\&D Systems) in 24-well plates pre-coated with $0.2 \mathrm{~mL}$ growth factor-reduced Matrigel (BD Biosciences, Bedford, MA). The number of capillary sprouts was counted in 10 fields per well. Data were obtained from triplicate wells per condition and are representative of at least 3 independent experiments. Photographs were taken at $\times 10$ magnification each day for 7 days.

\section{Tooth Slice/Scaffold Assay}

Tooth slices $(1.3 \mathrm{~mm}$ thick) were obtained from sound human third molars extracted at the Department of Oral Surgery (University of Michigan). The dental pulp was carefully removed, and scaffolds were prepared by using a poly-L-lactic/chloroform solution in the pulp chamber filled with sodium chloride ( $\mathrm{NaCl})(250-425 \mu \mathrm{m})$, as described previously (6). Specimens were treated with $10 \%$ EDTA for 1 minute. Six $\times$ $10^{5}$ transduced DPSCs were seeded in each tooth slice/scaffold $(\mathrm{n}=6$ per experimental condition) and transplanted subcutaneously into the dorsum of severe combined immunodeficient mice (CB.17 SCID; Charles River, Wilmington, MA) immediately after seeding. After 28 days, tooth slice/scaffolds were retrieved and fixed with $10 \%$ formaldehyde for 24 hours at $4^{\circ} \mathrm{C}$ and demineralized with Decalcifier II (Surgipath; Richmond, IL) for 24 hours at room temperature. Hematoxylin-eosin staining and immunohistochemistry with rabbit turbo EGFP (Bethyl Laboratories, Montgomery, TX) were used to evaluate the morphology and density of DPSC-derived microvessels. Vessels were counted in 10 fields per tooth slice/scaffold by a calibrated evaluator (intraclass correlation coefficient $=0.95$ ) blinded for experimental conditions. Institutional review boards approved the protocols regulating these studies.

\section{Results Signaling through LRP6 Is Necessary for Activation of Beta-Catenin in DPSCS}

To begin to define the relative contribution of different receptors on Wnt $/ \beta$-catenin signaling in DPSCs, we silenced LRP 6 or Frizzled 6 and exposed them to rhWnt1 or rhVEGF 165 (Fig. $1 A$ and $B$ ). We used several different shRNA sequences for gene silencing and used DPSCs transduced with the sequences that were most efficient in the experiments included here, ie, sequence \#1 for LRP6 and \#30 for Frizzled6 (Fig. 1A). As expected, the expression of active $\beta$-catenin was induced by Wnt1 or VEGF in a dose-dependent manner in DPSCs (Fig. 1C). In contrast, in LRP6-silenced DPSCs, active $\beta$-catenin was not upregulated by VEGF or Wnt1. Silencing Frizzled6 did not have a significant impact on VEGF-induced or Wnt1-induced $\beta$-catenin activation (Fig. $1 C$ ).

\section{LRP6 Signaling Is Necessary for Vasculogenic Differentiation of DPSES In Vitro}

Here we defined the impact of LRP6 and Frizzled 6 on the expression of endothelial markers in DPSCs. In shRNA-control DPSCs, the endothelial differentiation medium (ie, EGM2-MV supplemented with $\mathrm{VEGF} \pm$ Wnt1) induced expression of the endothelial marker VEGFR2 and VEGFR1 (Fig. 2A). The same was observed with Frizzled6-silenced DPSCs (Fig. 2A). In contrast, LRP6-silenced DPSCs did not express endothelial markers in response to the endothelial differentiation medium (Fig. 2A). Interestingly, both VEGF and IL-8 were constitutively downregulated in LRP6-silenced DPSCs when compared with control DPSCs (Fig. $2 B$ and $C$ ).
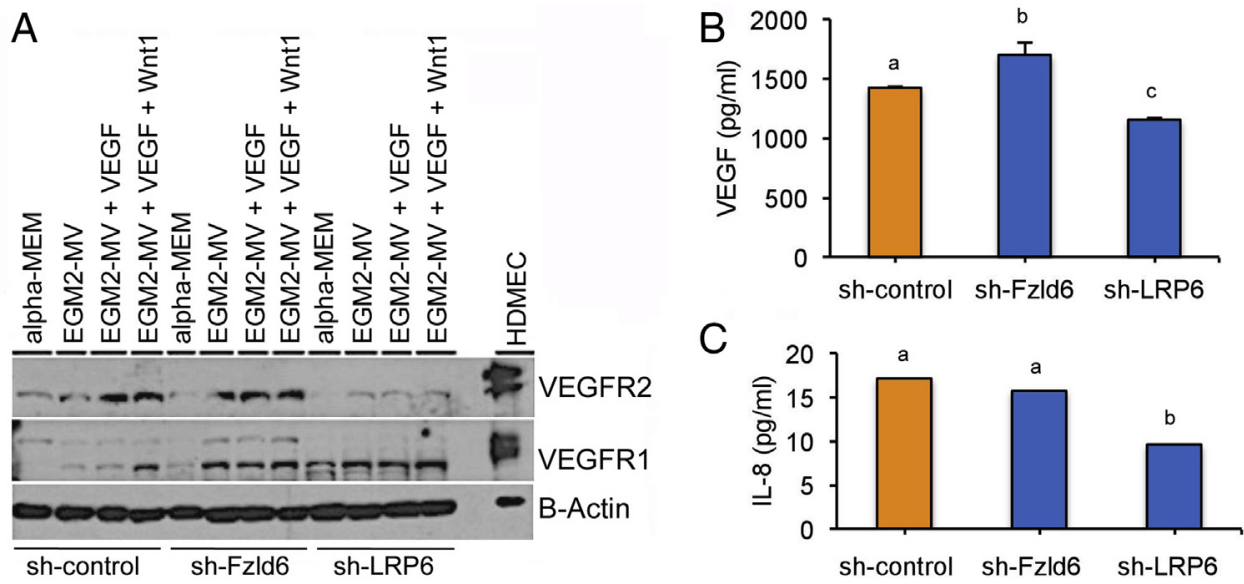

Figure 2. LRP6 silencing inhibits vasculogenic differentiation of DPSCs in vitro. (A) Western blots depicting protein expression of endothelial markers (VEGFR1, VEGFR2) on culture of DPSCs in control medium (alpha-MEM), endothelial growth medium (EGM2-MV), or EGM2-MV supplemented with $50 \mathrm{ng} / \mathrm{mL}^{\mathrm{rhVEGF}} 165$ or rhWnt1. $(B$ and $C$ ) Graphs depicting results of ELISA for VEGF $(B)$ and IL-8 $(C)$ in DPSCs cultured in control alpha-MEM medium. Different lower case letters represent $P<.05$. 
Capillary sprouting assay in Matrigel was used to analyze the impact of Wnt receptors on the vasculogenic potential of DPSCs in vitro. We observed that endothelial cells (HDMECs) quickly formed capillary sprouts in Matrigel within a few hours. In contrast, DPSCs took much longer to differentiate into endothelial cells and form capillary sprouts (Fig. 3A). However, DPSC-shRNA-LRP6 and DPSC-shRNAFrizzled6 showed a trend for lower numbers of capillary sprouts compared with vector control DPSCs, but the differences were not significant (Fig. 3B).

\section{LRP6 Silencing Inhibits Endothelial Differentiation of DPSES In Vivo}

To verify our in vitro results, DPSCs were seeded in tooth slices/ scaffolds and implanted in the subcutaneous space of immunodeficient mice. Twenty-eight days after transplantation, tooth slices/scaffolds were retrieved, and pulp-like tissues were observed in the pulp chambers (Fig. 4A). Because all our shRNA lentiviruses contained an EGFP cassette, we performed immunohistochemistry for this exogenous marker as a strategy to quantify the number of DPSC-derived (human) blood vessels. We observed that there were fewer EGFP-positive blood vessels $(P<.05)$ in the pulps engineered with LRP6-silenced DPSCs, as compared with Frizzled6-silenced DPSCs or empty vector control cells (Fig. 4B).

\section{Discussion}

It is known that DPSCs have ability to differentiate into multiple cells lineage (4-6); they are a viable source for dental pulp tissue engineering (4). We have demonstrated that human DPSCs are capable to differentiate into endothelial cells that organize themselves into functional blood vessels that connect with the host vasculature $(5,6)$. We have also shown that VEGF and Wnt1 trigger signaling events that result in the differentiation of DPSCs into endothelial cells (5). However, we did not know which Wnt receptor was responsible for the vasculogenic differentiation of endothelial cells.

We have previously shown that dental pulp stem cells of exfoliated teeth (SHED) do not express endothelial markers (ie, CD31, VEGFR2) when cultured in control alpha-MEM (21). However, when SHED cells are exposed to EGM2-MV medium supplemented with hhVEGF $_{165}$, they differentiated into endothelial cells (21). Here we report similar trends for DPSCs retrieved from permanent teeth. It is known that Wnt $\beta$ - catenin signaling plays an important role in vasculogenesis (22). Because VEGF expression can be upregulated by activation of the Wnt pathway (10), it is plausible that autologous VEGF can induce angiogenesis $(6,21,23)$. Also, Wnt1 was found to directly promote proliferation, tube formation, and induction of the proangiogenic IL-8 transcription in human endothelial cells $(11,22)$. $\beta$-catenin is the key mediator of the canonical Wnt signaling. This signaling pathway is activated when Wnt ligands bind to a member of the Frizzled family and a member of LRP family of co-receptors. We found that Frizzled3, 4, 5, and 6 and LRP5 and 6 are expressed in DPSCs (data not shown). Here we decided to focus on Frizzled6 and LRP6 because these receptors were consistently highly expressed in DPSCs.

Interestingly, we noticed that active $\beta$-catenin expression was higher in LRP6-silenced DPSCs and in Frizzled6-silenced DPSCs treated with rhVEGF $_{165}$ or rhWnt1. These results are in line with the fact that one of the functions of LRP 6 is the inhibition of the $\beta$-catenin destruction complex through direct inhibition of GSK3- $\beta$ activity. However, it is important to notice that $\beta$-catenin and GSK3- $\beta$ participate in other signaling pathways (eg, AKT signaling) or as a structural component of cellular adhesion complex (24-26). In addition, LRP6 and Frizzled6 also play a role as negative regulators of $\mathrm{Wnt} / \beta$-catenin signaling through TCF/LEF $(25,27)$. Our data suggest that LRP6 and Frizzled6 also regulate the activity of $\beta$-catenin amount in postnatal stem cells (eg, DPSCs).

We observed that the angiogenic potential of Frizzled6-silenced DPSCs is similar to control cells in most of the experiments performed here. Silencing of Frizzled6 does not interfere with the transcription of VEGFR1 and VEGFR2 that play critical roles in the endothelial differentiation of DPSCs (6). In contrast, LRP6-silenced DPSCs did not show upregulation of VEGFR2 in response to the endothelial differentiation medium. These data suggest that LRP6 plays a critical role in the regulation of the angiogenic potential in DPSCs. This might be related to the observation that LRP6 is required for the activation of TCF/LEF activity $(28,29)$.

It has been reported that undifferentiated DPSCs expressed soluble proangiogenic factors such as VEGF and IL-8 (30). Notably, LRP6silenced DPSCs showed lower expression levels of VEGF and IL-8 than vector control cells. This observation might provide additional mechanistic explanation for the lower vasculogenic activity that was found in LRP6-silenced cells in vitro and in vivo. We observed that LRP6silenced DPSCs transplanted into SCID mice generated fewer blood
A
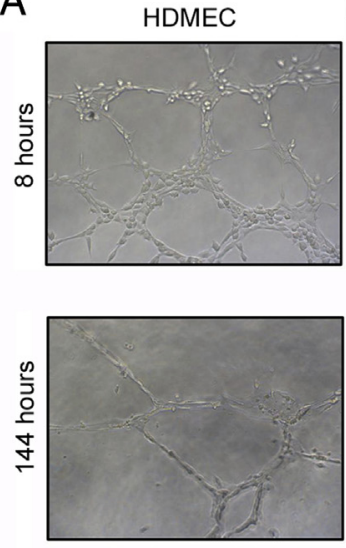

DPSC-shRNA-Frizzled-6 (144 h)
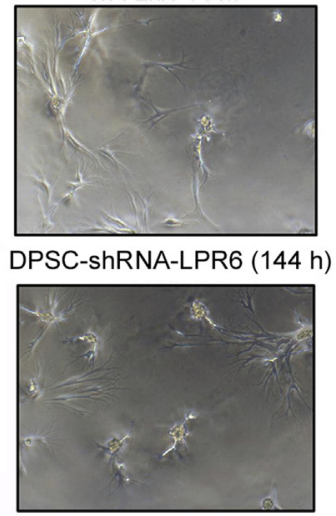

B

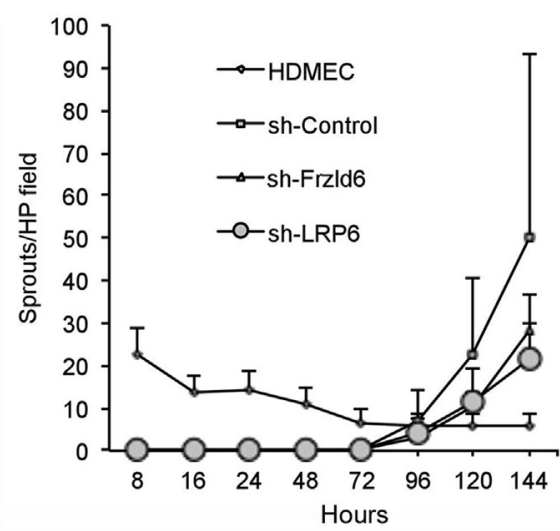

Figure 3. Effect of LRP6 or Frizzled6 signaling on capillary sprouting of DPSCs in three-dimensional cultures. (A) Photomicrographs of capillary tube networks generated by HDMECs or by DPSCs seeded in Matrigel and exposed to EGM2-MV supplemented with $50 \mathrm{ng} / \mathrm{mL} \mathrm{rhVEGF}_{165}$ for up to 144 hours (original magnification, $\times 200)$. (B) Graph depicting number of sprouts/microscopic field generated by HDMECs, DPSC-silenced cells, or controls seeded on Matrigel and stimulated with EGM2-MV supplemented with $50 \mathrm{ng} / \mathrm{mL} \mathrm{rhVEGF}_{165}$ for up to 144 hours. 
A

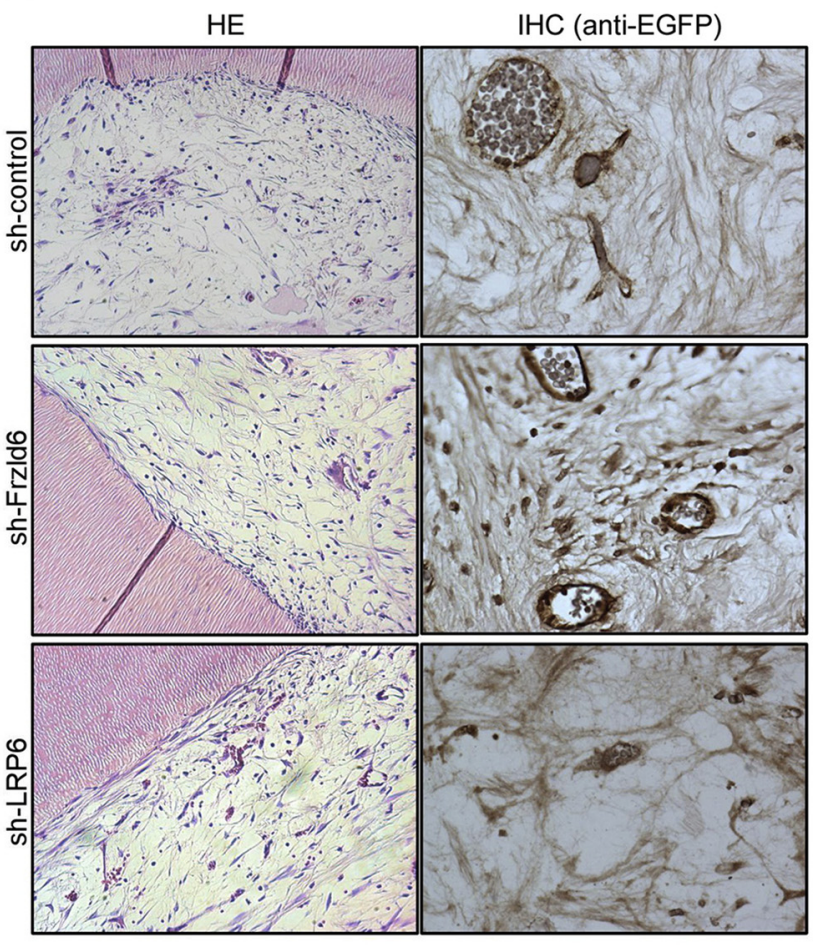

B

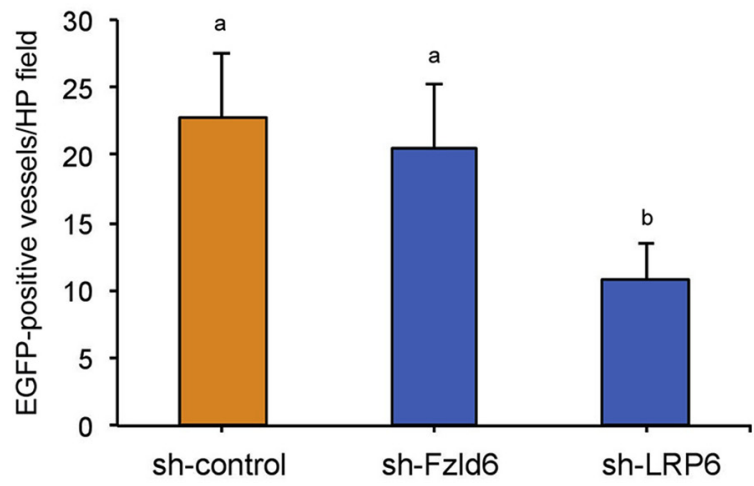

Figure 4. LRP6 silencing inhibits endothelial differentiation of DPSCs in vivo. (A) Tooth slices/scaffolds seeded with LRP6-silenced DPSCs, Frizzled6-silenced DPSCs, or vector control DPSCs were transplanted into the subcutaneous space of immunodeficient mice. After 28 days, tooth slice/scaffolds were retrieved, fixed, and analyzed by hematoxylin-eosin (HE) staining (original magnification, $\times 200$ ) or immunohistochemistry (IHC) with EGFP antibody (original magnification, $\times 400) .(B)$ Graph depicting density of EGFP-positive blood vessels (ie, generated by DPSCs) in 6 tooth slice/scaffolds per experimental condition. Blood vessels were counted in 10 microscopic fields/specimen at $\times 200$ magnification. Different lower case letters represent $P<.05$.

vessels, when compared with vector control DPSCs. In contrast, the pulps generated with Frizzled6-silenced DPSCs showed similar vascularization as compared with pulps generated with control DPSCs. These data confirm our in vitro observations and demonstrate a critical role for Wnt signaling in the determination of the vasculogenic fate of DPSCs. These data also suggest the possibility of directing the fate of DPSCs toward non-vasculogenic phenotypes on targeted inhibition of LRP6.

In conclusion, our data confirmed the hypothesis that $\mathrm{Wnt} / \beta$ catenin pathway induces the vasculogenic differentiation of DPSCs and unveiled a new role for LRP6 signaling in this process. These data align nicely with the seminal observation that the $\mathrm{Wnt} / \beta$-catenin pathway inhibits odontoblastic differentiation of DPSCs (17). We propose that the $\mathrm{Wn} t / \beta$-catenin pathway serves as a "switch" regulating the fate of DPSCs between odontoblastic/osteoblastic and vasculogenic. Such knowledge could be exploited in translational dental pulp tissue engineering. For example, one could enforce activation of the $\mathrm{Wnt} / \beta$ catenin pathway immediately after injection of DPSCs in the root canal to maximize the rapid generation of functional blood vessels $(5,6)$ that will bring the oxygen and nutrients required to maintain cell viability and function in the critical first days after transplantation. This could be potentially achieved by intracanal delivery of microspheres for controlled release of rhWnt1 for a period that would likely not be longer than 7-10 days. Once a vascular network is established, the exogenous Wnt-1 would no longer be necessary, and DPSCs would be primed to respond to dentin-derived factors (eg, bone morphogenetic protein 2) that will induce their differentiation into functional odontoblasts $(6,31)$. We propose that a deep understanding of processes involved in the regulation of DPSC fate will enable the development of a mechanism-based therapeutic strategy for the engineering of a functional dental pulp tissue in the treatment of necrotic immature permanent teeth.

\section{Acknowledgments}

This work was funded by grant R01DE21410 from NIH/NIDCR (J.E.N.) and by grant 2012/13039-0 and 2012/24244-3 from FAPESP (G.O.S.).

Publication of this supplement was supported by Septodont. The authors deny any conflicts of interest related to this study.

\section{References}

1. Nakashima M, Akamine A. The application of tissue engineering to regeneration of pulp and dentin in endodontics. J Endod 2005;31:711-8.

2. Nör JE. Tooth regeneration in operative dentistry. Oper Dent 2006;31:633-42.

3. Brey EM, Uriel S, Greisler HP, McIntire LV. Therapeutic neovascularization: contributions from bioengineering. Tissue Eng 2005;11:567-84.

4. Gronthos S, Brahim J, Li W, et al. Stem cell properties of human dental pulp stem cells. J Dent Res 2002;81:531-5.

5. Zhang $\mathrm{Z}$, Nör $\mathrm{F}$, Oh $\mathrm{M}$, et al. Wnt/ $\beta$-catenin signaling determines the vasculogenic fate of post-natal mesenchymal stem cells. Stem Cells 2016;34:1576-87.

6. Sakai VT, Zhang Z, Dong Z, et al. SHED differentiate into functional odontoblasts and endothelium. J Dent Res 2010;89:791-6.

7. Nelson WJ, Nusse R. Convergence of Wnt, beta-catenin, and cadherin pathways. Science 2004;303:1483-7.

8. Cadigan KM, Nusse R. Wnt signaling: a common theme in animal development. Genes Dev 1997;11:3286-305.

9. Reya T, Clevers H. Wnt signalling in stem cells and cancer. Nature 2005;434: $843-50$.

10. Zhang X, Gaspard JP, Chung DC. Regulation of vascular endothelial growth factor by the Wnt and K-ras pathways in colonic neoplasia. Cancer Res 2001;61:6050-4.

11. Masckauchán TN, Shawber CJ, Funahashi Y, et al. Wnt/beta-catenin signaling induces proliferation, survival and interleukin-8 in human endothelial cells. Angiogenesis 2005;8:43-51.

12. Lindsley RC, Gill JG, Kyba M, et al. Canonical Wnt signaling is required for development of embryonic stem cell-derived mesoderm. Development 2006;133:3787-96.

13. Nichols AS, Floyd DH, Bruinsma SP, et al. Frizzled receptors signal through G proteins. Cell Signal 2013;25:1468-75.

14. Jernigan KK, Cselenvi CS, Thorne CA, et al. Gbetagamma activates GSK3 to promote LRP6-mediated beta-catenin transcriptional activity. Sci Signal 2010;3:ra37.

15. Maretto S, Cordenonsi M, Dupont S, et al. Mapping Wnt/beta-catenin signaling during mouse development and in colorectal tumors. Proc Natl Acad Sci U S A 2003; 100:3299-304.

16. Ye X, Wang Y, Cahill H, et al. Norrin, frizzled-4, and Lrp5 signaling in endothelial cells controls a genetic program for retinal vascularization. Cell 2009;139:285-98.

17. Scheller EL, Chang J, Wang CY. Wnt/beta-catenin inhibits dental pulp stem cell differentiation. J Dent Res 2008;87:126-30. 


\section{Regenerative Endodontics}

18. Silvério $\mathrm{KG}$, Davidson $\mathrm{KC}$, James $\mathrm{RG}$, et al. Wnt/ $\beta$-catenin pathway regulates bone morphogenetic protein (BMP2)-mediated differentiation of dental follicle cells J Periodontal Res 2012;47:309-19.

19. Feng $X$, Xing J, Feng G, et al. Age-dependent impaired neurogenic differentiation capacity of dental stem cell is associated with Wnt/ $\beta$-catenin signaling. Cell Mol Neurobiol 2013;33:1023-31.

20. Gronthos S, Mankani M, Brahim J, et al. Postnatal human dental pulp stem cells (DPSCs) in vitro and in vivo. Proc Natl Acad Sci U S A 2000;97:13625-30.

21. Bento LW, Zhang Z, Imai A, et al. Endothelial differentiation of SHED requires MEK1/ ERK signaling. J Dent Res 2013;92:51-7.

22. Wright M, Aikawa M, Szeto W, Papkoff J. Identification of a Wnt-responsive signal transduction pathway in primary endothelial cells. Biochem Biophys Res Commun 1999:263:384-8.

23. Mullane EM, Dong Z, Sedgley CM, et al. Effects of VEGF and FGF2 on the revascularization of severed human dental pulps. J Dent Res 2005;87:1144-8.

24. Zhang J, Shemezis JR, McQuinn ER, et al. AKT activation by N-cadherin regulates beta-catenin signaling and neuronal differentiation during cortical development. Neural Dev 2013;8:7.
25. Golan T, Yaniv A, Bafico A, et al. The human Frizzled 6 (HFz6) acts as a negative regulator of the canonical Wnt/beta-catenin signaling cascade. J Biol Chem 2004; 279:14879-88.

26. McCrea PD, Turck CW, Gumbiner B. A homolog of the armadillo protein in Drosophila (plakoglobin) associated with E-cadherin. Science 1991;254: 1359-61.

27. Beagle B, Johnson GV. Differential modulation of TCF/LEF-1 activity be the soluble LRP6-ICD. PLoS One 2010;5:e11821.

28. Beagle B, Mi K, Johnson GV. Phosphorylation of PPP(S/T)P motif of the free LRP6 intracellular domain is not required to activate the Wnt/betacatenin pathway and attenuate GSK3beta activity. J Cell Biochem 2009;108:886-95.

29. Wu G, Huang $\mathrm{H}$, Abreu JG, He X. Inhibition of GSK3 phosphorylation of $\beta$-catenin via phosphorylated PPSPXS motifs of Wnt coreceptor LRP6. PLoS One 2009; $4:$ e4926.

30. Bronckaers A, Hilkens P, Fanton Y, et al. Angiogenic properties of human dental pulp stem cells. PLoS One 2013;8:e71104.

31. Casagrande L, Demarco F, Zhang Z, et al. Dentin-derived BMP2 and odontoblastic differentiation of SHED. J Dent Res 2010;89:603-8. 